\title{
An Experimental Configuration to Probe for Lorentz Symmetry Violation in Electrons Using Trapped $\mathbf{Y b}^{+}$Ions
}

\author{
Noah Schlossberger*, Phil Richerme \\ Department of Physics, Indiana University Bloomington
}

*Corresponding Author email: nkschlos@iu.edu

Article History

Received: 22 February 2018

Revised: 31 March 2018

Accepted: 1 April 2018

Published: 04 April 2018

Student(s)

- Noah Schlossberger

Academic Year: 2017-2018, Fall Sem

Course Level: Bachelor

Course Name: B.S. (Applied Physics)

Course year: $3^{\text {rd }}$ Year, $\mathrm{VI}^{\text {th }}$ Semester

Mentor(s)

- $\quad$ Phil Richerme

\begin{abstract}
Since extensions of the standard model have been developed that predict violations of local Lorentz invariance (LLI), precision measurement groups have been working to reduce experimental bounds of the associated matrix element. Using an analogue of the Michelson-Morley test with trapped $\mathrm{Ca}+$ ions, the current bound has been set at one part in $10^{18}$. However, by instead using $\mathrm{Yb}+$ ions, which have highly stable electronic states for storing quantum information compared to their counterparts and exhibit enhanced effects of LLI breaking asymmetries, we can push the bounds to one part in 1023. In this article, we outline a configuration for such an experiment and offer solutions to experimental concerns. We develop an algorithm for state creation, manipulation, and measurement that minimizes measurement time and transition uncertainty. We also discuss necessary hardware for trapping and manipulating ions including a vacuum system, a Paul trap and the associated electrode voltage supplies, and an optics system for generating and applying transition pulses. The experiment is specifically designed to utilize the existing ion trap hardware in place at the Richerme lab at Indiana University Bloomington.
\end{abstract}

Keywords : LLI ; Trapped Ion ; Tabletop Experiment ; Lorentz Symmetry Violation ; Standard Model Extension

\section{Introduction}

Lorentz symmetry is a staple characteristic of modern physics, but recent developments in theory predict the possibility of violations in local Lorentz invariance (LLI). The Standard-Model Extension, for example, allows for the breaking of Lorentz and charge-parity-time reversal (CPT) symmetries. This prompts the urgency of experimental measurements of predicted Lorentz violation effects. A recent experiment [1] using $\mathrm{Ca}^{+}$ions has set an upper limit for Lorentz-violation in electrons at the level of one part in $10^{18}$. However, the experiment suffered the limiting factors of short decay lifetimes of its target states and a limited effect of Lorentz invariance. Both of these limitations can be improved by choosing a long lasting state in Ytterbium ions that exhibits enhanced asymmetry effects, specifically the $4 \mathrm{f}^{13} 6 \mathrm{~s}^{2}{ }^{2} \mathrm{~F}_{7 / 2}$ state, which is stable with a lifetime of order 10 years and has a negative reduced matrix element $\left\langle J\left|T^{2}\right| J\right\rangle$ [2]. Though performing 
the experiment with $\mathrm{Yb}^{+}$ions has been proposed theoretically [2] there are still considerable issues to address, especially with regards to how to actually implement the experiment. This paper lays out a scheme for executing the experiment both in terms of experimental approach and hardware configuration.

\section{Experiment Scheme}

The experiment is an analogue of the Michelson-Morley experiment, which originally probed for the existence of an aether using an interferometer. In our iteration, however, Michaelson interferometry is replaced with atomic interferometry and the existence of an aether is replaced by the existence of a preferred reference frame. Two atoms are trapped in a linear Paul trap and a uniform magnetic field is applied in laboratory $\hat{z}$. The ions are placed into a decoherence free subspace of two entangled states. The system then oscillates in time on the Bloch sphere with a frequency corresponding to the energy difference between the states. As the magnetic field and ion system rotate with respect to the sun's rest frame, LLI breaking will cause an oscillation in this energy, and thus the frequency of the Bloch sphere oscillation will itself oscillate in time. By taking a series of measurements of identically prepared entangled states, varying the time the system is allowed to oscillate, and repeating, we can find the period of oscillation, which corresponds to the energy difference between the two entangled states. If the LLI breaking matrix elements are nonzero, we should observe variations in this frequency throughout the day. Each individual measurement must then be made quickly enough to be short compared to the daily cycle of laboratory frame rotation.

\subsection{LLI Asymmetry Effects}

Because the induced magnetic field of the experiment is perpendicular to the surface of the earth, the field is then mostly perpendicular to the tangential of the lab frame rotation (with only an angular difference of the laboratory's offset from the rotational equator). This gives us interferometric effect we are looking for. We are looking for LLI breaking asymmetries in the form of perturbations to the Hamiltonian, resulting in a shift in the energy difference between atomic states. This energy shift is given by [1]

$\Delta E_{L L I}=\boldsymbol{Q} \cdot C_{0}^{(2)}(t)$

where $\mathcal{Q}=\frac{1}{h}\left(\Delta E_{0}\right), \quad \Delta E_{0}$ is the energy difference between the states used in the experiment, and $C_{0}^{(2)}=-3 \sin (2 \chi) c_{X Z} \cos \left(\omega_{E} t\right)-3 \sin (2 \chi) c_{Y Z} \sin \left(\omega_{E} t\right)-\frac{3}{2}\left(c_{X X}-c_{Y Y}\right) \sin ^{2}(\chi) \cos \left(2 \omega_{E} t\right)-$ $3 c_{X Y} \sin ^{2}(\chi) \sin \left(2 \omega_{E} t\right)$

Here, $\omega_{E}$ is the earth's angular velocity $=2 \pi(23.93)$ hours, and $\chi$ is the colatitude of the laboratory. In Bloomington, Indiana (home of the Richerme laboratory), the colatitude $\chi=50.83^{\circ}$.

The $c_{i j}$ coefficients in Eq. (2) represent matrix elements in the electron sector which can be directly mapped to the photon sector by [1]

$\left\{\begin{aligned} c_{X Y} & =\frac{1}{2} \tilde{\kappa}_{e-}^{X Y} \\ c_{X Z} & =\frac{1}{2} \tilde{\kappa}_{e-}^{X Z} \\ c_{Y Z} & =\frac{1}{2} \tilde{\kappa}_{e-}^{Y Z} \\ c_{X X}-c_{Y Y} & =\frac{1}{2}\left(\tilde{\kappa}_{e-}^{X X}-\tilde{\kappa}_{e-}^{Y Y}\right)\end{aligned}\right.$

where $\tilde{\kappa}$ are parameters of the Standard-Model Extension (SME) Lagrangian shift for electromagnetic fields [1]

$\delta \mathcal{L}=\frac{1}{2}\left[\vec{E} \cdot \tilde{\kappa}_{e-} \cdot \vec{E}-\vec{B} \cdot \tilde{\kappa}_{e-} \cdot \vec{B}\right]$

If we can measure the energy shift throughout the day as given by Eq. (1) and bound the coefficients in $C_{0}^{(2)}$, then we can bound the Standard Model Extension's LLI-violating Lagrangian perturbation given by Eq. (4) with the mapping from Eq. (3). 
Schlossberger et al., Adv. J. Grad. Res.; Vol. 4, Issue 1, pp: 15-33, July 2018

\subsection{Creation of a Decoherence Free Subspace}

Fig. (1) shows the relevant energy level diagram for ${ }^{171} \mathrm{Yb}^{+}$. For this experiment, we will be working with states in the $4 \mathrm{f}^{13} 6 \mathrm{~s}^{2}{ }^{2} \mathrm{~F}_{7 / 2}$ level (labeled $\left.(F=3)^{2} F_{7 / 2}\right)$.

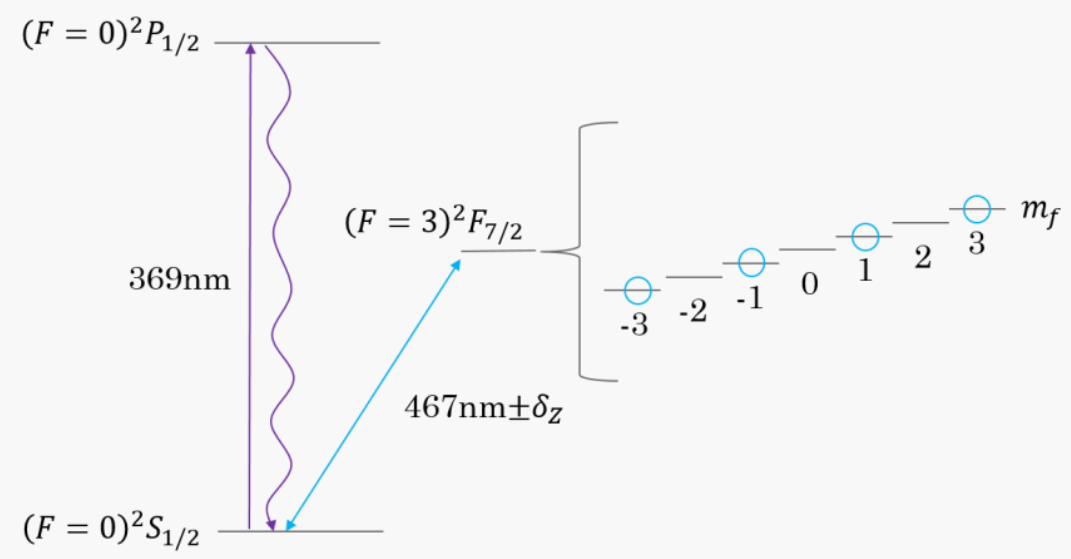

Figure 1: Energy level diagram for ${ }^{171} \mathrm{Yb}^{+}$

The level is split in the presence of the applied magnetic field according to the total angular momentum. While [2] works with eigenstates of $m_{j}$ (which are equivalent to $m_{f}$ for even isotopes of $\mathrm{Yb}$ ), this does not take into account the nuclear spin $I=\frac{1}{2}$ created by the extra neutron in ${ }^{171} \mathrm{Yb}^{+}$. Instead we work with the $J+I$ coupled basis $F$ and label our states by the secondary total angular momentum quantum number

$$
\left\{\begin{array}{l}
\left.| \pm 1\rangle=\left.\right|^{2} F_{7 / 2} ; m_{f}= \pm 1\right\rangle \\
| \pm 3\rangle=\left|{ }^{2} F_{7 / 2} ; m_{f}= \pm 3\right\rangle
\end{array}\right.
$$

(We are not concerned with Fermi/Bose statistics of integer-momentum states because we are dealing with non-fundamental particles that are physically separated enough as not to be subject to exchange statistics). We start out by placing our ions into a set of antisymmetric states with one ion in a superposition of $m_{f}=$ $+1,+3$ and the other in the superposition $m_{f}=-1,-3$. To distinguish which ion is assigned the positive or negative superposition, we name the states $\left|\Psi_{L}\right\rangle$ and $\left|\Psi_{R}\right\rangle$ with the subscript indicating which ion is given the positive mixed state (Fig. 2).

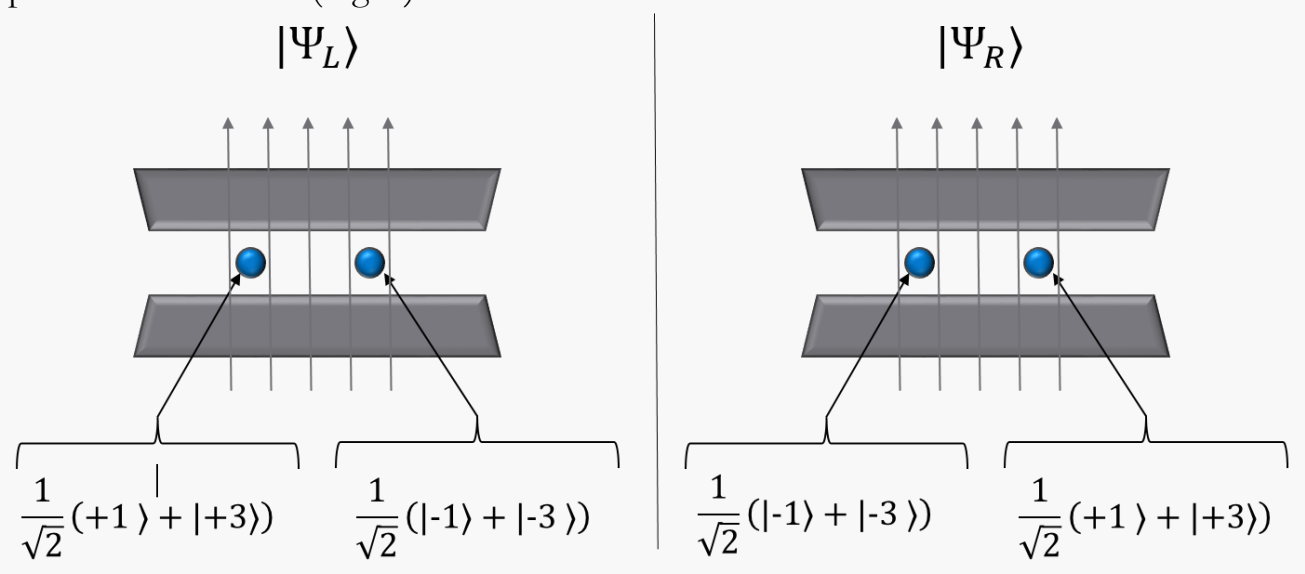

Figure 2: The initial state layout for the experiment

The product state of the two ions in this configuration is then

$\left|\Psi_{L, R}^{P}\right\rangle=\frac{1}{\sqrt{2}}(| \pm 1\rangle+| \pm 3\rangle) \otimes \frac{1}{\sqrt{2}}(|\mp 1\rangle+|\mp 3\rangle)$

which can be expanded as a coupled state

$$
\left|\Psi_{L, R}^{P}\right\rangle=\frac{1}{2}(| \pm 1, \mp 1\rangle+| \pm 3, \mp 3\rangle+| \pm 1, \mp 3\rangle+| \pm 3, \mp 1\rangle)
$$


An Experimental Configuration to Probe for Lorentz Symmetry Violation in Electrons Using Trapped $Y b^{+}$Ions

The presence of the magnetic field $B \hat{z}$ causes energy shifts given by

$$
\Delta U=\sum_{i} \mu_{i} B
$$

where $\mu_{i}$ is the magnetic moment of each ion. The overall energy shifts are then only nonzero for the $\mid \pm$ $1, \mp 3\rangle$ and $| \pm 3, \mp 1\rangle$ components. Because the applied magnetic field has some amount of random fluctuation and noise, this will cause decoherence in these states, resulting in a decoherence free subspace of the remaining unshifted states. After the unbalanced states are removed we are left with the entangled set of states

$\left|\Psi^{L, R}\right\rangle=\frac{1}{\sqrt{2}}(| \pm 1, \mp 1\rangle+| \pm 3, \mp 3\rangle)$

\subsection{Measurement}

Under the Schrödinger equation, the state given by Eq. (6) freely evolves according to

$\left|\Psi^{L, R}(t)\right\rangle=\frac{1}{\sqrt{2}}\left(| \pm 3, \mp 3\rangle+e^{i\left(\Delta E_{L, R} t / \hbar+\phi_{L, R}\right)}| \pm 1, \mp 1\rangle\right)$

This looks like precession about the equator of the Bloch sphere with the two entangled states as poles (Fig. 3).

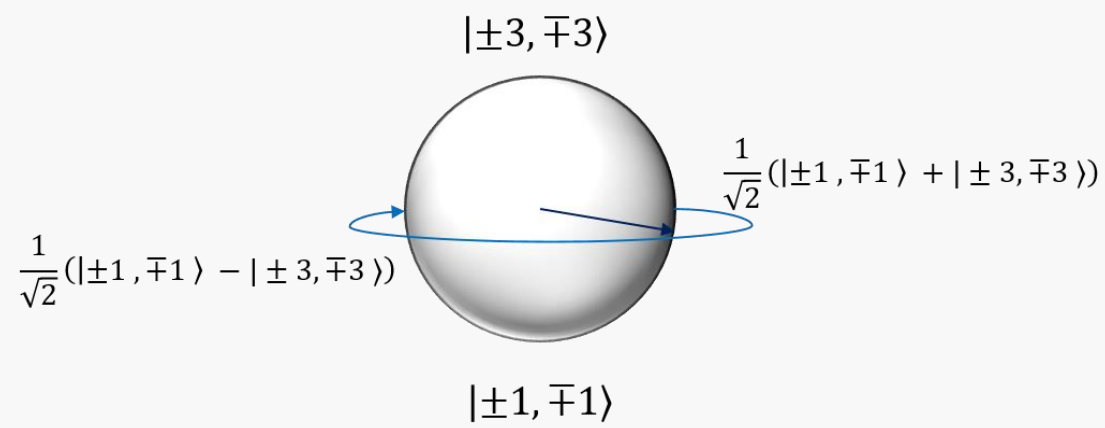

Figure 3: An illustration of the time evolution of the state in the form of precession on the Bloch sphere

The Bloch precession occurs at frequency

$\omega=\frac{\Delta E_{L, R}}{\hbar}$

where $\Delta E_{L, R}$ is the energy difference between the entangled states $| \pm 1, \mp 1\rangle$ and $| \pm 3, \mp 3\rangle$. Thus by measuring the frequency, we can find the energy difference between the states. To do this we can execute a scheme as follows: We prepare the entangled state given by Eq. (7), wait a Ramsey duration $T$, and measure the states from an eigenbasis

$$
\left\{\begin{array}{l}
\left|+_{L, R}\right\rangle=\frac{1}{\sqrt{2}}(| \pm 3, \mp 3\rangle+| \pm 1, \mp 1\rangle) \\
\left|-{ }_{L, R}\right\rangle=\frac{1}{\sqrt{2}}(| \pm 3, \mp 3\rangle-| \pm 1, \mp 1\rangle)
\end{array}\right.
$$

We can read from this eigenbasis by applying a $\frac{\pi}{2}$ pulse between $| \pm 3, \mp 3\rangle$ and $| \pm 1, \mp 1\rangle$ (which can be decomposed into individual ion transitions). This pulse acts as a unitary transformation from $\left\{\left|+_{L, R}\right\rangle,\left|-_{L, R}\right\rangle\right\}$ to $\{| \pm 3, \mp 3\rangle,| \pm 1, \mp 1\rangle\}$ (as shown in Appendix A.1). We can read from this basis by applying a $\pi$ pulse from $| \pm 1, \mp 1\rangle$ to the dual ground state $\left|(F=0)^{2} S_{\frac{1}{2}},(F=0)^{2} S_{\frac{1}{2}}\right| \equiv|0,0\rangle$, applying a fluorescence pulse, and imaging with a charge-coupled device (CCD) sensor to check for fluorescence. This works because the $\pi$ pulse will only work for ions initially in the $| \pm 1, \mp 1\rangle$ state and the fluorescence pulse will only work for ions in the ground state. Thus, our measurement algorithm looks like

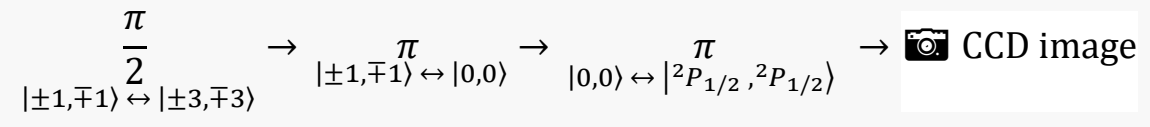


with results interpreted by

\begin{tabular}{|l|l|}
\hline Fluorescence Detected? & State \\
\hline Yes & $\left|+_{L, R}\right\rangle$ \\
No & $\left|-_{L, R}\right\rangle$ \\
\hline
\end{tabular}

By preparing the state from Eq. (7), waiting a time $T$, and measuring multiple times, we can find the probabilities $P_{+}(T)$ and $P_{-}(T)$ of finding the system in each of the $\left|+_{L, R}\right\rangle$ and $\left|-{ }_{L, R}\right\rangle$ states. Because $P_{+}=\left|\left\langle+_{L, R} \mid \Psi^{L, R}\right\rangle\right|^{2}=\left|\frac{1}{\sqrt{2}}(| \pm 3, \mp 3\rangle+| \pm 1, \mp 1\rangle) \frac{1}{\sqrt{2}}\left(| \pm 3, \mp 3\rangle+e^{i\left(\frac{\Delta E_{L, R} T}{\hbar}+\phi_{L, R}\right)}| \pm 1, \mp 1\rangle\right)\right|^{2}=$ $\left|\frac{1}{2}\left(1+e^{i\left(\frac{\Delta E_{L, R} T}{\hbar}+\phi_{L, R}\right)}\right)\right|^{2}=\frac{1}{2}-\frac{1}{2} \cos \left(\frac{\Delta E_{L, R} T}{\hbar}+\phi_{L, R}\right)$

and similarly

$P_{+}=\left|\left\langle-_{L, R} \mid \Psi^{L, R}\right\rangle\right|^{2}=\left|\frac{1}{\sqrt{2}}(| \pm 3, \mp 3\rangle-| \pm 1, \mp 1\rangle) \frac{1}{\sqrt{2}}\left(| \pm 3, \mp 3\rangle+e^{i\left(\frac{\Delta E_{L, R} T}{\hbar}+\phi_{L, R}\right)}| \pm 1, \mp 1\rangle\right)\right|^{2}=$ $\left|\frac{1}{2}\left(1-e^{i\left(\frac{\Delta E_{L, R} T}{\hbar}+\phi_{L, R}\right)}\right)\right|^{2}=\frac{1}{2}+\frac{1}{2} \cos \left(\frac{\Delta E_{L, R} T}{\hbar}+\phi_{L, R}\right)$

Then taking the difference gives

$$
P_{+}-P_{-}=\cos \left(\frac{\Delta E_{L, R} T}{\hbar}+\phi_{L, R}\right)
$$

Which is a simple sinusoid at the frequency we want to measure. By then varying $T$ and repeating, we can measure the angular frequency $\omega=\frac{\Delta E_{L, R}}{\hbar}$ and extract the energy difference. Each time we do this, we can take a separate measurement for both the $\left|\Psi_{L}\right\rangle$ and $\left|\Psi_{R}\right\rangle$ configurations and average the results. This will cancel effects due to any magnetic field gradients that might exist in the experiment that would otherwise affect the data. We then take many such series of measurements throughout the day to find $\omega(t)$ and fit the results to the expected form $\omega(t)=\omega_{0}\left(1+C_{0}^{(2)}(t)\right)$. This will allow us to extract the $c_{i j}$ coefficients from our fitted $C_{0}^{(2)}(t)$ by comparing our fit to Eq. (2).

\section{Procedures for State Manipulation}

\subsection{Mixed State Creation}

In order to prepare the product state given by Eq. (5), we start with each ion in the ground state ${ }^{2} \mathrm{~S}_{1 / 2}(\mathrm{~F}=0)$ $\equiv|0\rangle$. Let the transition on the left ion be indicated with subscript $L$ and on the right with subscript $R$. With both ions in the initial ground state, we apply the following series of pulses:

$$
\begin{aligned}
& |0\rangle \otimes|0\rangle \\
& \underset{\substack{\frac{\pi}{2} \\
|0\rangle \leftrightarrow|1\rangle}}{\longrightarrow} \frac{1}{\sqrt{2}}(|0\rangle+i|1\rangle) \otimes|0\rangle \\
& \underset{\substack{\pi_{L} \\
|0\rangle \leftrightarrow|3\rangle}}{\longrightarrow} \frac{1}{\sqrt{2}}(|3\rangle+|1\rangle) \otimes|0\rangle \\
& \underset{\substack{\frac{\pi}{2} \\
\mid}}{|0\rangle \leftrightarrow|1\rangle} \frac{1}{\sqrt{2}}(|3\rangle+|1\rangle) \otimes \frac{1}{\sqrt{2}}(|0\rangle+i|1\rangle) \\
& \underset{\substack{\pi_{R} \\
|0\rangle \leftrightarrow|3\rangle}}{\longrightarrow} \frac{1}{\sqrt{2}}(|3\rangle+|1\rangle) \otimes \frac{1}{\sqrt{2}}(|3\rangle+|1\rangle)
\end{aligned}
$$

This gives us $\left|\Psi_{L}^{P}\right\rangle$ as in Eq. (5), and we can construct $\left|\Psi_{R}^{P}\right\rangle$ under exchange of $L$ and $R$ operations. 


\subsection{Rabi Durations}

For transitions to be feasible for our experiment they must have $\pi$ pulse durations $T_{\pi}$ that are low enough to not impede the time resolution of the experiment but long enough to be within the capabilities of our hardware. To calculate $T_{\pi}$, we begin with the Rabi frequency, given by [3]

$$
\Omega=\frac{E_{0}}{\hbar}\left\langle\psi_{1}|e x| \psi_{2}\right\rangle
$$

where $\left|\psi_{1}\right\rangle$ and $\left|\psi_{2}\right\rangle$ are the states with respectively lower and higher energy and $E_{0}$ is the magnitude of the electric field due to the driving light pulse (assuming that the laser is linearly polarized along laboratory $\hat{x}$ ). We need to know the magnitude of the dipole matrix element as well as the electric field magnitude. The Einstein coefficient is given by [3]

$$
A_{21}=\frac{\omega_{0}^{3}}{3 \pi \epsilon_{0} \hbar c^{3}}\left|\left\langle\psi_{1}|\operatorname{er}| \psi_{2}\right\rangle\right|^{2}=\frac{1}{\tau}
$$

where $\omega_{0}$ is the atomic transition frequency. Because we have a singular beam linearly polarized in $\hat{x}$, we can take $\boldsymbol{r} \rightarrow x$. We can then extract the dipole matrix element magnitude

$$
\left|\left\langle\psi_{1}|e x| \psi_{2}\right\rangle\right|=\sqrt{\frac{3 \pi \epsilon_{0} \hbar c^{3}}{\tau \omega_{0}^{3}}}
$$

Next, since the intensity of the beam is given by

$$
I=\frac{1}{2} c \epsilon_{0}\left|E_{0}\right|^{2}
$$

We can express the electric field magnitude in terms of the beam intensity

$$
\left|E_{0}\right|=\sqrt{\frac{2 I}{c \epsilon_{0}}}
$$

Combining our expressions,

$$
\Omega=\frac{\sqrt{\frac{2 I}{c \epsilon_{0}}}}{h} \sqrt{\frac{3 \pi \epsilon_{0} \hbar c^{3}}{\tau \omega_{0}^{3}}}=\sqrt{\frac{6 \pi I c^{2}}{\hbar \tau \omega_{0}^{3}}}
$$

The $\pi$ pulse period is then given by

$T_{\pi}=\frac{\pi}{\Omega}=\sqrt{\frac{\pi \hbar \tau \omega_{0}^{3}}{6 I c^{2}}}$

\subsection{7nm Baseline Transition}

The Zeeman variations of the $467 \mathrm{~nm}$ transition will be difficult because the $S_{1 / 2}$ to $F_{7 / 2}$ transition is an electric octupole transition and therefore forbidden by standard selection rules. The experimental value of $\omega_{0}$ for this transition in the absence of a uniform magnetic field has been found [4] to be $2 \pi \times 642121496772645.15(52) H z$ with fractional uncertainty $7.1 \times 10^{-17}$. The state lifetime $\tau$, however, is not well known; it is experimentally bounded as $5.4_{-3.6}^{+9.3}$ years [5] and a theoretical analysis puts it at 8.4 years [6]. While this long lifetime gives us the extended measurement time we are looking for, it also significantly impedes the speed with which we can complete the transition. Using the theoretical value, we can estimate Eq. (11)

$T_{\pi} \approx \frac{103.4}{\sqrt{I}} S$

where $I$ is the beam intensity in $\mathrm{W} / \mathrm{m}^{2}$. Ignoring signal loss in the optical path and assuming perfect incidence, a general formula for the intensity of a Gaussian beam of radius $r$ is

$$
I=\frac{2 P}{\pi r^{2}}
$$


For a $12 \mathrm{~mW}$ beam of radius $20 \mu \mathrm{m}$ (the configuration used in [4]), we would have a $\pi$ pulse duration

$$
T_{\pi} \approx 24 m s
$$

This time period is easily within the bounds of what is experimentally executable and is in order-ofmagnitude agreement with the period used to drive the transition in [4]. However, in the context of this experiment this is a long time, making this transition the rate limiting step in how fast we can take successive measurements.

In order for Eq. (11) to hold and accordingly for the transition to be reasonably fast, the width of the laser must be significantly less than the (power broadened) width of the transition. As the transition width is around $6.6 \mathrm{~Hz}$ for $.5 \mathrm{~mW}$ focused laser power [4], we then require the laser width to be of unitary order in Hz. One possible method of doing so is to stabilize the transition beam with a Fabry-Pérot cavity (Fig. 4).

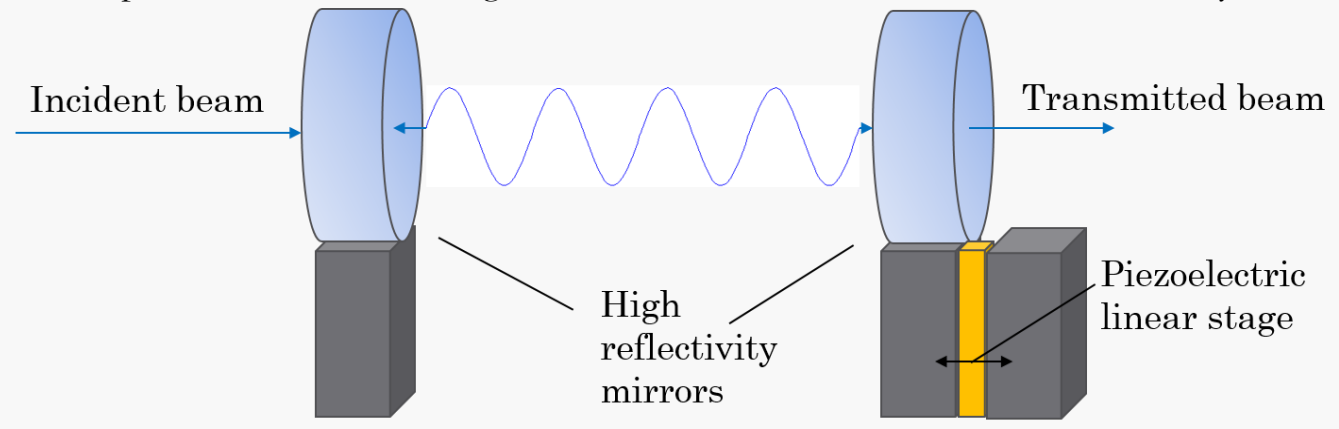

Figure 4: Layout of the Fabry-Pérot cavity

The cavity is a pair of concave mirrors separated by an integer number of wavelengths, with one mirror on a piezoelectric stage such that this distance can be finely tuned. Laser frequencies that are off resonance will reflect on a non-node point in the phase of the beam, which will continue to reflect at different phases eventually destructively interfering and effectively cancelling out. On resonance signals, however, will constructively interfere. Because off resonance signals will only hit the output mirror several times before signal loss but on resonance signals will continue to reflect back and forth until transmittance occurs, the transmitted light will be almost entirely on resonance.

\section{$3.4 \quad{ }^{2} D_{5 / 2}$ as an Intermediary State}

Another option for the state transition is to first transition to the ${ }^{2} \mathrm{D}_{5 / 2}$ state by the $411 \mathrm{~nm}$ line, and then back down to the desired ${ }^{2} \mathrm{~F}_{7 / 2}$ state through the $3.4 \mu \mathrm{m}$ line. The linewidths of these transitions are significantly less narrow than for the direct $467 \mathrm{~nm}$ transition, so if laser linewidth is a limiting factor, this could be a more favorable option. Because the transition is an electric dipole transition, the Ramsey $\pi$ duration is relatively small. The frequency of the ${ }^{2} \mathrm{D}_{5 / 2}-{ }^{2} \mathrm{~F}_{7 / 2}$ transition is measured [7] to be $2 \pi \times 87362471500(800) \mathrm{kHz}$ with a natural linewidth of $22 \mathrm{~Hz}$.

\subsection{Ensuring State Transition}

During the application of our Ramsey pulse, the probability of the atom being in the $\mathrm{F}_{7 / 2}$ state oscillates in time as

$$
P_{F_{7 / 2}}(t)=\sin ^{2}\left(\frac{\Omega t}{2}\right)
$$

We can experimentally find the configuration-specific $\Omega$ by taking our rough estimate for $T_{\pi}$ from Eq. (12) and varying slightly, each time taking several measurements of the state until maximum coupling is achieved. Once we know $T_{\pi}$ to some experimental certainty, we can then drive the transition to the $\mathrm{F}_{7 / 2}$ state at such a certainty. To significantly reduce this uncertainty, we can employ another transition, a 369nm fluorescence pulse, to the ion. Since this will only cause the ion to fluoresce if the ion is in the S state and not in the F state, the action of applying the pulse and imaging with CCD constitutes a measurement that will collapse the wavefunction. Thereby, we can execute the initial excitation algorithm described in Fig. 5. 


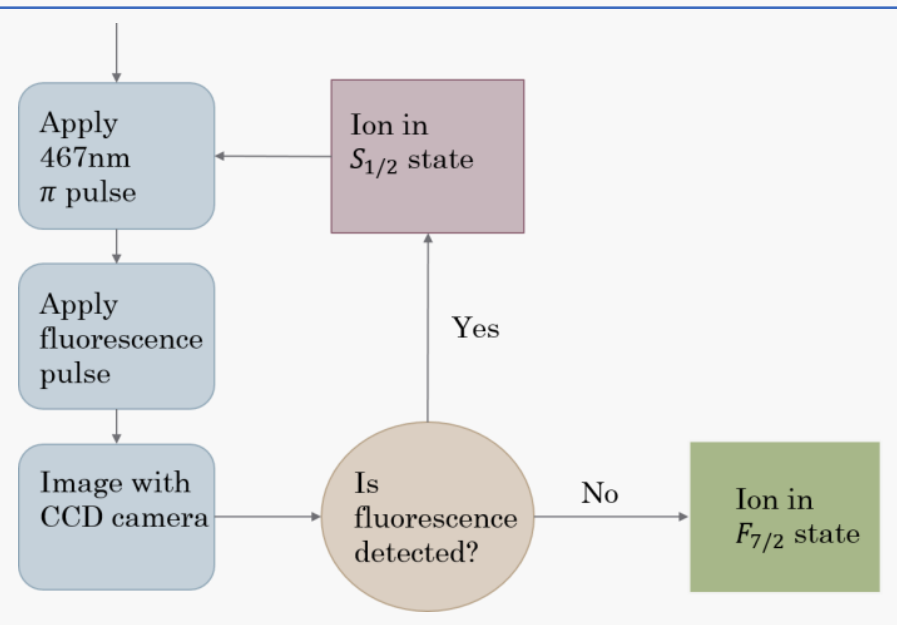

Figure 5: The algorithm for placing the ions into the $F_{7 / 2}$ state

However, by requiring imaging we potentially require a longer time to prepare the measurement state, as the camera triggering, capturing, processing, and logic evaluation time is of similar magnitude to the pulse duration of the transition. Since we need very fine time resolution for the experiment due to the repetitive measurements needed to extract probability, we may choose to omit this step. The Rabi period of the transition $(\sim 24 \mathrm{~ms})$ is greater than the imaging time $(\sim 10 \mathrm{~ms})$ so the time increase of this step is of order unity.

\subsection{Sublevels of the $F_{7 / 2}$ Level}

The Zeeman shift for the $(\mathrm{F}=3)^{2} \mathrm{~F}_{7 / 2}$ level due to the magnetic field is given by [3]

$$
\Delta E=-\mu B=-g_{F} \mu_{B} m_{f} B
$$

where $\mu_{B}$ is the Bohr magneton and $g_{F}$ is the gyromagnetic ratio, given to first order as

$$
g_{F}=g_{J} \frac{F(F+1)-I(I+1)+J(J+1)}{2 F(F+1)}
$$

Here,

$$
g_{J}=\frac{3}{2}+\frac{S(S+1)-L(L+1)}{2 J(J+1)}
$$

And $S$ is the Total spin principal quantum number $(=1 / 2), L$ is the Total angular orbital momentum principal quantum number $(=3)$, I is the Total nuclear spin principal quantum number $(=1 / 2), J$ is the spinorbital principal quantum number $(=7 / 2)$, and $F$ is the total angular momentum principal quantum number $(=3)$. For the states in question we find

$$
g_{F}=1.2857 \rightarrow \Delta E=1.1924 \times 10^{-23} \Delta m_{f} B
$$

The energy frequency relationship is

$$
E=\hbar \omega \rightarrow \Delta \omega=\frac{1}{\hbar} \Delta E
$$

So the change in angular frequency for a transition between two Zeeman states is

$\Delta \omega=2 \pi \times \Delta m_{f} B \cdot 17.995 \mathrm{GHz}$

where $B$ is the experiment magnetic field in Tesla (which we will take to be $3.930 \times 10^{-4}$ to be consistent with the calculations in the original [2] proposal). Then the energy differences within the $\mathrm{F}_{7 / 2}$ state lead to transition frequency shifts

$$
\left\{\begin{array}{l}
|0\rangle \leftrightarrow| \pm 1\rangle, \Delta m_{f}= \pm 1, \Delta \omega= \pm 2 \pi \times 7.053 \mathrm{MHz} \\
|0\rangle \leftrightarrow| \pm 3\rangle, \Delta m_{f}= \pm 3, \Delta \omega= \pm 2 \pi \times 21.16 \mathrm{MHz}
\end{array}\right.
$$


Schlossberger et al., Adv.J. Grad. Res.; Vol. 4, Issue 1, pp: 15-33, July 2018

\section{Experiment-specific Hardware Layout}

The hardware functionalities needed to complete the experiment are a functional ion trap, the ability to generate a magnetic field perpendicular to the surface of the Earth, and the ability to generate and trigger the laser pulses that drive the state transitions of the experiment.

\subsection{Paul trap components}

The Paul trap uses a series of blades with electric potentials to confine the ions. On the radial blades, RF potentials are applied to produce an electric potential of the form of a rotating saddle point, which confines the ions radially. On the axial blades, DC potentials are applied which create an axial potential minimum that confines the ions axially. The configuration is shown in Fig. 6

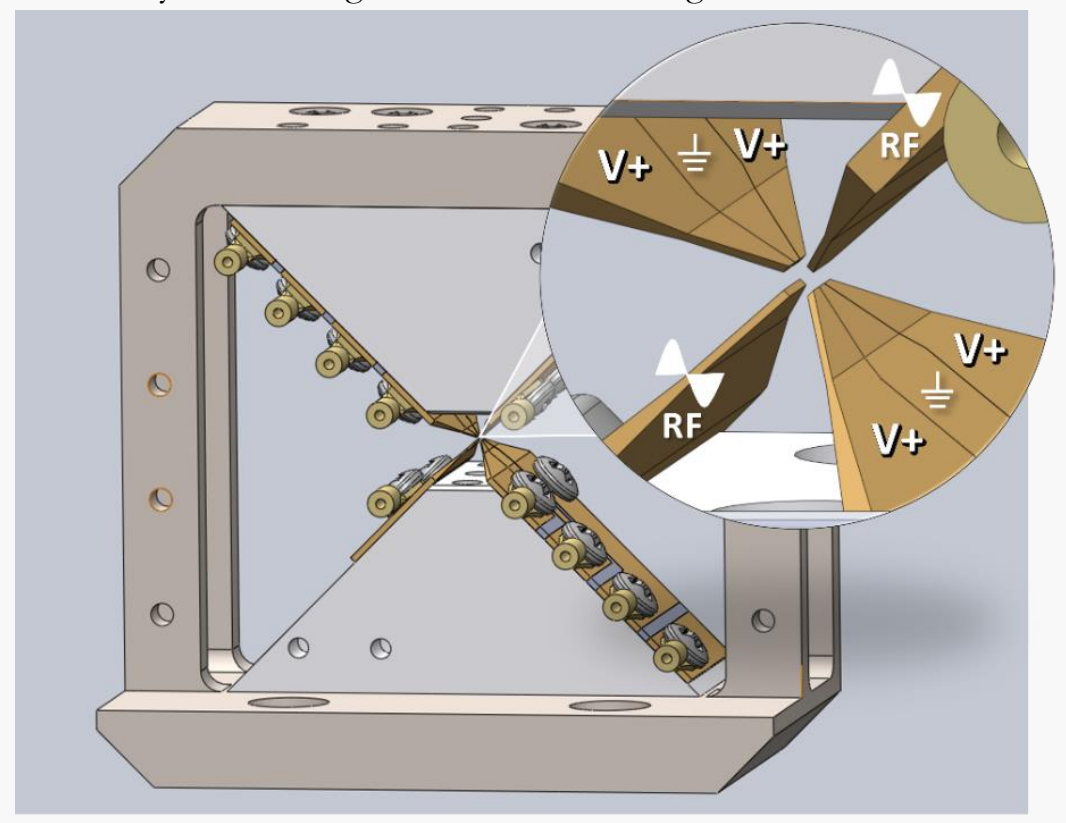

Figure 6: The Paul trap blade configuration for applying electric potentials

\subsubsection{Vacuum Chamber}

In order to prevent interaction between the ions and the background gas and to ensure the quantum system is isolated, we need to remove enough air from the chamber that the mean collision period between trapped ions and background gas is much greater than the state preparation and measurement period.

$$
\tau_{\text {collision }} \gg \tau_{\text {measurement }}
$$

An order of magnitude calculation is appropriate to find the required pressure for our experiment. We begin with an approximation for the collision time. For a more conservative estimate we will take into account heating interactions that could disrupt the quantum information of the system rather than just the Langevin collisions. Our collision rate is then given by [8]

$\tau_{\text {collision }}=\frac{1}{\lambda}=\frac{1}{n\langle\sigma v\rangle} \approx \frac{1}{n \pi \Gamma\left(\frac{1}{3}\right)}\left[\frac{16 \epsilon_{0} h}{a q^{2}}\right]^{2 / 3}\left\langle v^{-1 / 3}\right\rangle$

where $q$ is the electron charge and $a$ is the Van der Waals coefficient.

Inside the vacuum chamber the primary gas remaining will be $H_{2}$ gas, so we approximate the background gas as consisting entirely of $\mathrm{H}_{2}$. Assuming room temperature, we can treat the background as an ideal gas, where

and

$$
\left\langle v^{-1 / 3}\right\rangle \approx\left[\frac{3 k T}{m}\right]^{-1 / 6}
$$

$$
P=n k T
$$


By substituting these into Eq. (15), we find

$P \approx \frac{k T}{\tau_{\text {collision }} \pi \Gamma\left(\frac{1}{3}\right)}\left[\frac{16 \epsilon_{0} h}{a q^{2}}\right]^{2 / 3}\left[\frac{3 k T}{m}\right]^{-1 / 6}$

At room temperature, this gives us

$$
\tau_{\text {collision }}>100 s \rightarrow P<10^{-10} \text { Torr }
$$

To accomplish this, we use Ultra High Vacuum (UHV) components, with which we can reduce the pressure to such a value. The vacuum configuration is shown in Fig. 7.

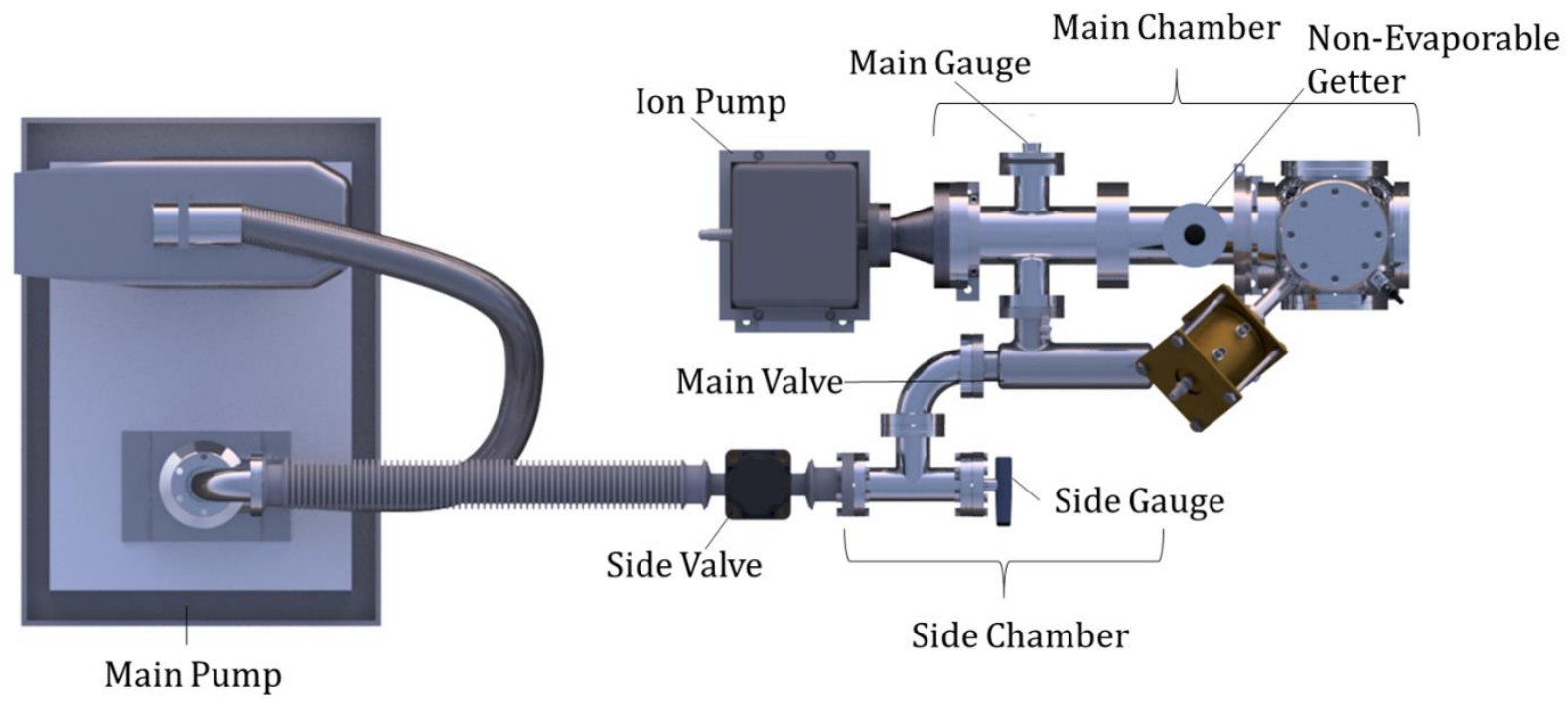

Figure 7: The vacuum layout of the experiment for achieving $U H V$

First we use an Agilent IDP3 vacuum pump to reduce the whole system to around $10^{-6}$ Torr. We then close the valve between the main chamber and the side chamber and the valve between the side chamber and the main pump. We then use the Agilent Starcell ion pump to reduce pressure to $10^{-9}$, and finally the SAES st172 non-evaporable getter to bring the chamber to sub-10-10 Torr. The purpose of the side chamber is to act as a buffer stage when breaking vacuum so that we can slowly reduce pressure in a multistage fashion.

\subsubsection{Helical Resonator}

The Paul trap requires a high voltage, low noise alternating current supply at a very narrow frequency. To do this we use a traditional waveform generator and a helical resonator, a cavity with a pair of concentric coils coupled to a cylindrical shell to create a high gain, high quality factor, and low noise amplifier. The dimensions of the coil are labeled in Fig. 8.

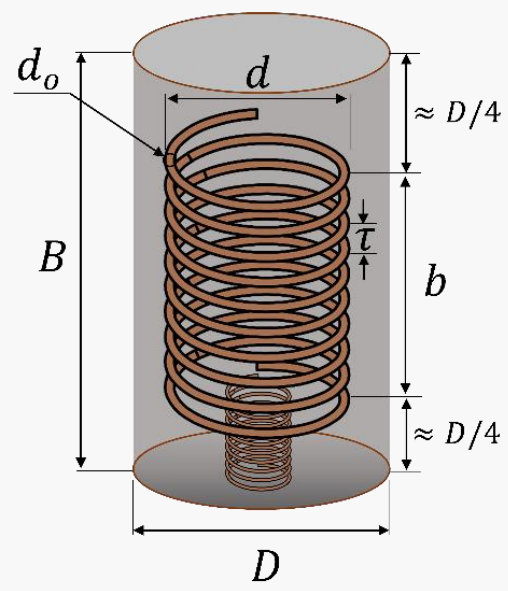

Figure 8: Dimensional labeling and layout for the helical resonator 
The copper pipe we use has inner diameter $D=7.62 \mathrm{~cm}$, and our main coil will use $10 \mathrm{AWG}$ wire $\left(d_{0}=\right.$ $2.58826 \mathrm{~mm}$ ). We are free to select the rest of the dimensions such that we maximize the quality factor $\mathcal{Q}$ of the resonator and match the resonant frequency $\omega_{0}$ to the needed RF frequency, given the constraints

$$
\left\{\begin{array}{c}
b \leq B-\frac{D}{2} \\
\frac{b}{d} \geq 1
\end{array}\right.
$$

In our case we want $\omega_{0}=2 \pi \times 50 \mathrm{MHz}$. The resonant frequency is given by [9]

$\omega_{0}=\frac{1}{\sqrt{\left(C_{S}+C_{t}+C_{w}+C_{C}\right) L_{C}}}$

where $C_{C}$ is the self-capacitance of the main coil, given by [9]

$$
C_{C} \approx\left(\frac{11.26 b}{d}+8+\frac{27}{\sqrt{\frac{b}{d}}}\right) d \times 10^{-12} \mathrm{~F}
$$

(where dimensions are in meters), $C_{s}$ is the capacitance between the coil and the shield, given by [9]

$$
C_{s} \approx b \cdot 39.37 \frac{3}{4 \log (\mathrm{d} / \mathrm{D})} \times 10^{-12} \mathrm{~F}
$$

$L_{C}$ is the inductance of the main coil, given by [9]

$$
L_{C}=b\left(39.37 \frac{0.025 d^{2}\left(1-\left(\frac{d}{D}\right)^{2}\right)}{\tau^{2}} \times 10^{-6}\right) \mathrm{H}
$$

and $C_{\omega}$ and $C_{t}$ are the capacitances of the wiring between the trap and the resonator and of the trap itself, which are measured quantities. The quality factor which we wish to optimize is given by [9]

$\mathcal{Q}=\frac{X_{L_{C}}}{\left(\frac{R_{C} X_{C_{C}}^{2}}{R_{C}^{2}+\left(X_{C_{C}}+X_{L_{C}}\right)^{2}}+\frac{R_{t} X_{R}^{2}}{R_{t}^{2}+\left(X_{R}+X_{T}\right)^{2}}+R_{S}+R_{j}\right)}$

where $R_{t}$ is the measured resistance of the trap, $R_{c}$ is the resistance of the helical coil, $X_{R}$ is the equivalent reactance due to the capacitance between the coil and surrounding shield as well as between the ion trap and connecting wires, $X_{C_{C}}$ is the reactance due to the coil's self inductance, $X_{L_{C}}$ is the reactance due to the antenna coil's self inductance, and $X_{T}=X_{R} \frac{C_{S}+C_{\omega}}{C_{t}}$.

In the limit of $X_{L_{C}} \ll X_{C_{C}}, \quad R_{C} \ll X_{C_{C}}$, and $R_{T} \ll\left(X_{C_{t}}+X_{C_{\omega}}+X_{C_{s}}\right)$, which is the case with a low resistance coil material and proper connections, we can approximate the quality factor by [9]

$\mathcal{Q} \approx \frac{X_{L_{C}}}{R_{j}+R_{C}+R_{S}+R_{t}\left(\frac{\frac{X_{R}}{X_{T}}}{\frac{X_{R}}{X_{T}+1}}\right)}$ 
So our problem becomes

$$
\max _{\mathrm{B}, \mathrm{d}, \tau} \mathcal{Q}_{\text {(Eq. 19) }} \text { s.t. }\left\{\begin{array}{c}
d \geq d_{0} \\
\tau \geq d_{0} \\
b=B-\frac{D}{2} \\
\frac{b}{d} \geq 1 \\
\omega_{0_{\text {(Eq. 19) }}}=2 \pi \times 50 \mathrm{MHz}
\end{array}\right.
$$

Due to the complexity of our functions for $\mathcal{Q}$ and $\omega_{0}$, analytic optimization is not possible. Instead, discrete numerical optimization can be done by iterating over a range of values of $B, d$, and $\tau$ that satisfy the first four conditions, throwing away values such that $\left|\omega_{0}-2 \pi \times 50 \mathrm{MHz}\right| \geq 1 \mathrm{MHz}$, and finding the combinations such that $\mathcal{Q}$ has the greatest value. To minimize the resistance, capacitance, and noise in the lead wires between the coil and the trap, we mount the trap directly on the vacuum feedthrough (Fig. 9).

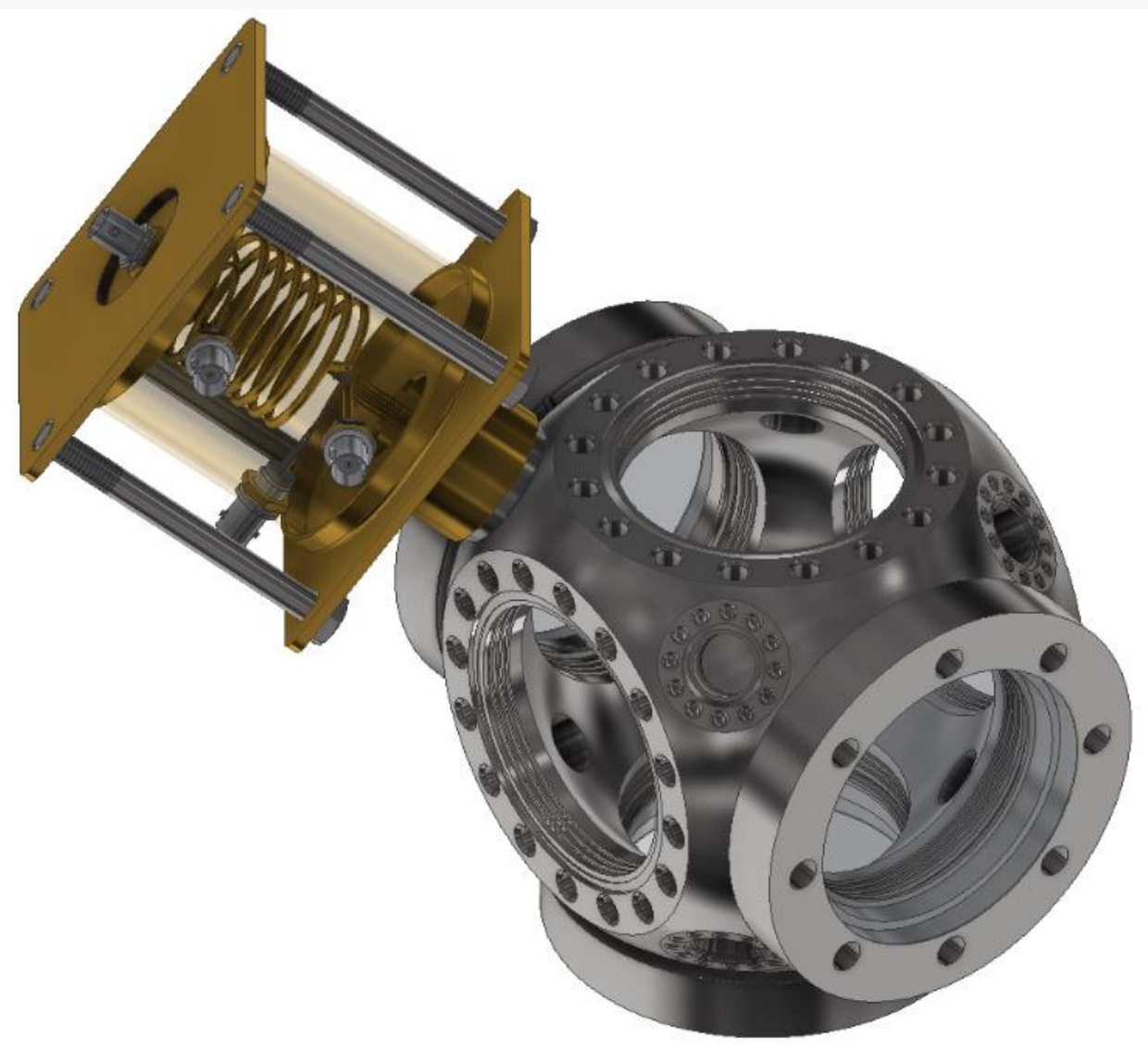

Figure 9: The helical resonator mounted directly on the vacuum feedthrough

\subsubsection{Electrodes}

To provide the DC voltages to the electrodes (labeled V+ in Fig. 6), we must have a way to set specific voltages from our control program. We design a system that uses a digital analog converter (National Instruments 9263 modules) to produce a base voltage in the $\pm 10 \mathrm{~V}$ range and operational amplifiers (Apex PA340CC) to scale the voltage to the $\pm 100 \mathrm{~V}$ range. The schematic for the op-amp board is shown in Fig. 10. 


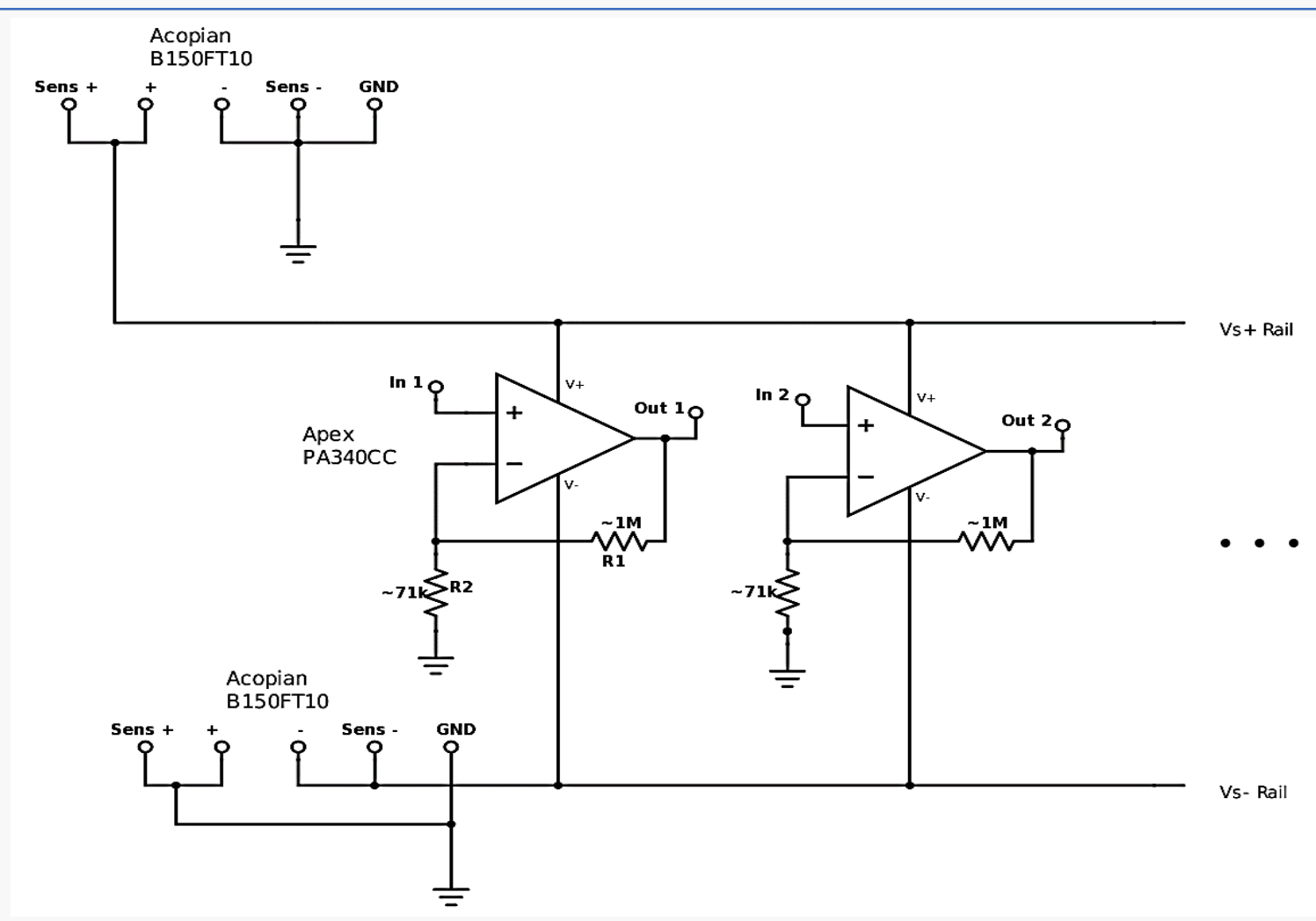

Figure 10: A schematic of the op-amp board for voltage scaling

The gain on each channel is given by

$$
G=1+\frac{R 1}{R 2} \sim 15
$$

Due to the artifacts and nonlinearities in both the National Instruments module and the operational amplifiers, to ensure exact calibration we generate a sweep of voltages with the NI, record the output of the op-amps, and perform a fit on the results. Inverting the fit, we have a function for the voltage to supply with the NI to produce the desired output voltage.

Because the signals will travel some finite distance between the circuit board and the vacuum feedthrough, there is a possibility that the lines will pick up some electromagnetic interference. To address this, we can place a low pass filter on each line just before the feedthrough, inline with the cable. An RLC circuit depticted in (Fig. 11) is appropratte, with a cutoff frequency of $10 \mathrm{~Hz}$.

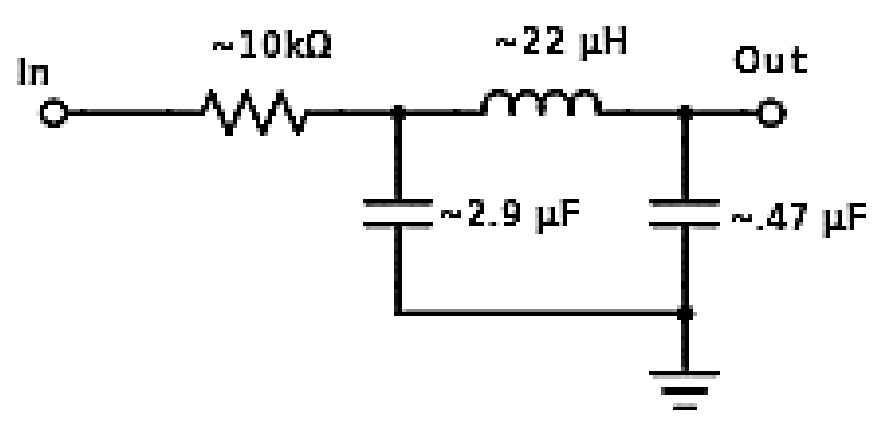

Figure 11: A schematic of the low-pass filter on the cable assembly

\subsection{Magnetic Field Generation}

A necessary component of the experiment is a stable laboratory $\hat{z}$-aligned magnetic field. To generate this, we use current carrying coils in each dimension. We need to cancel out any existing background fields and produce the uniform experiment field, so the generated field must be

$\vec{B}_{\text {gen }}=-\vec{B}_{\text {background }}^{\text {measured }}+\vec{B}_{z}^{\text {experiment }}$ 
An Experimental Configuration to Probe for Lorentz Symmetry Violation in Electrons Using Trapped $Y b^{+}$Ions

In our trap, the center is offset form the origin along the laboratory $y$-axis. This means that the field from the $y$-axis coil will have solely $-\hat{y}$ components, but the $\mathrm{x}$-axis coil will have $-\hat{x}$ and $+\hat{y}$ components, and similarly the $z$-axis coil will have $-\hat{z}$ and $+\hat{y}$ components (Fig. 12).

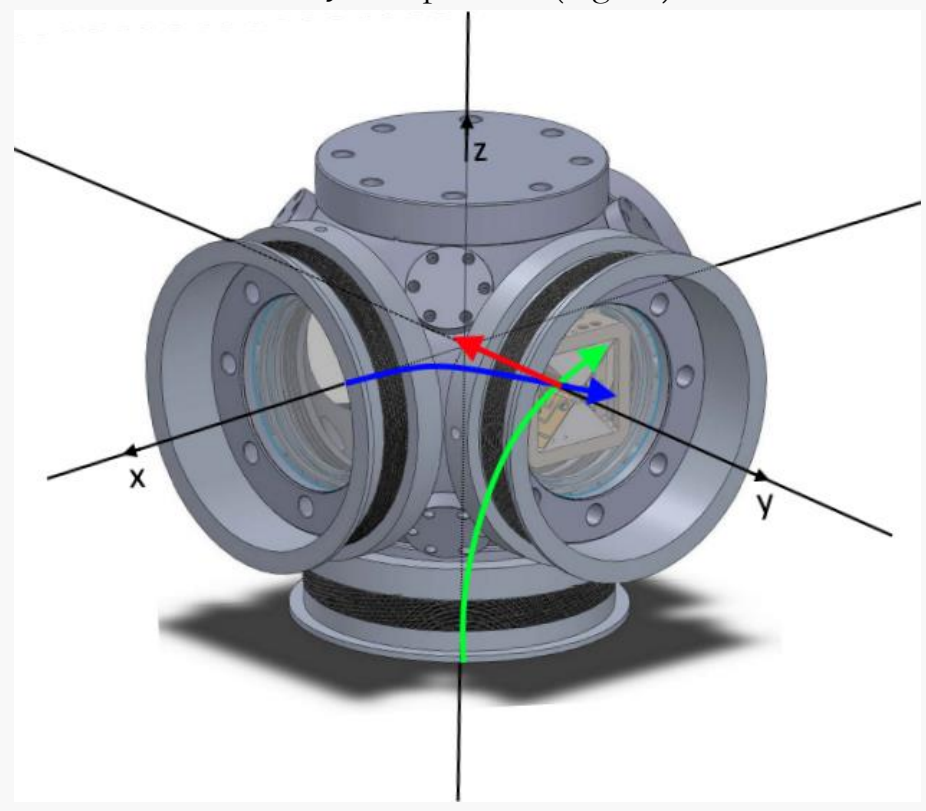

Figure 12: A diagram of the field inducing coil and trap configuration. The relevant magnetic field lines from each coil to the trap origin are depicted. The green arrow living in the z-y plane depicts the z-axis coil contribution, the blue arrow living in the $x$ - $y$ plane depicts the $x$-axis coil contribution, and the red arrow living on the $y$-axis depicts the $y$-axis coil contribution.

Let us begin by looking at the coil in $\hat{z}$. The coil can be approximated as a series of concentric loops of current, each at a different axial position and each layer having a different radial diameter. The magnetic field off-axis at the trap center from a single loop of wire is given by [10]

$$
\begin{aligned}
\vec{B}=\frac{\mu_{o} I a^{2} \cos \theta}{2\left(a^{2}+r^{2}\right)^{3 / 2}} & {\left[1+\frac{15 a^{2} r^{2} \sin ^{2} \theta}{4\left(a^{2}+r^{2}\right)^{2}}+\cdots\right] \hat{r} } \\
& -\frac{\mu_{o} I a^{2} \sin \theta}{4\left(a^{2}+r^{2}\right)^{5 / 2}}\left[2 a^{2}-r^{2}+\frac{15 a^{2} r^{2} \sin ^{2} \theta\left(4 a^{2}-3 r^{2}\right)}{8\left(a^{2}+r^{2}\right)^{2}}+\cdots\right] \hat{\theta}
\end{aligned}
$$

where $a$ is the radius of the loop, $\vec{r}$ is the vector from the loop origin to the trap center, and $\theta$ is the angle between the loop axis and the trap center. Re-expressing in Cartesian coordinates and using the fact that for our trap the $\mathrm{y}$-axis displacement is fixed $\left(\equiv y_{0}\right)$, we can write for the $\mathrm{z}$ axis coil

$$
\begin{aligned}
& \vec{B}(z, a)=\frac{\mu_{o} I a^{2}}{2\left(a^{2}+z^{2}+y_{0}^{2}\right)^{\frac{3}{2}}} \frac{z}{\left(y_{0}^{2}+z^{2}\right)}\left[1+\frac{15 a^{2} \sqrt{z^{2}+y_{0}^{2}} \frac{y_{0}^{2}}{y_{0}^{2}+z^{2}}}{4\left(a^{2}+r^{2}\right)^{2}}+\cdots\right]\left(y_{0} \hat{y}+z \hat{z}\right)- \\
& \frac{\mu_{o} I a^{2}}{4\left(a^{2}+z^{2}+y_{0}^{2}\right)^{\frac{5}{2}}} \frac{y_{0}}{\left(y_{0}^{2}+z^{2}\right)}\left[2 a^{2}-z^{2}-y_{0}^{2}+\frac{15 a^{2} y_{0}^{2}\left(4 a^{2}-3\left(z^{2}+y_{0}^{2}\right)\right)}{8\left(a^{2}+z^{2}+y_{0}^{2}\right)^{2}}+\cdots\right]\left(z \hat{y}-y_{0} \hat{z}\right)
\end{aligned}
$$

Letting $d_{w}$ be the diameter of the wire, $n_{t}$ be the number of turns per layer, $n_{l}$ be the number of layers, $z_{0}$ and $a_{0}$ be the minimum distance and radius, we can express the total magnetic field at the trap center due to the $z$-axis coil as a summation of the contributions from each loop

$\vec{B}_{\text {tot }}^{\mathrm{z} \text { coil }}=\sum_{i=0}^{n t-1} \sum_{\mathrm{j}=0}^{n l-1} \vec{B}\left(z_{0}+\left(i+\frac{1}{2}\right) d_{w}, a_{0}+\left(j+\frac{1}{2}\right) d_{w}\right)$

Using the formulation of $\vec{B}(z, a)$ from Eq. (22). A similar calculation can be done for the $\hat{x}$ - aligned coil by replacing $z, \hat{z}$, and $z_{0}$ with $x,-\hat{x}$, and $x_{0}$ respectively. Note that we must negate the unit vector because the $\mathrm{x}$-axis coil is located on the axis on the $x>0$ side whereas, the $z$-axis coil is located on the axis on the $z<0$ side as defined by our geometric convention. 
Schlossberger et al., Adv. J. Grad. Res.; Vol. 4, Issue 1, pp: 15-33, July 2018

Finally, calculating the field due to the y-axis coil is much simpler because it will be on-axis with the trap center. Such a field due to a single loop is given by [10]

$\vec{B}^{\text {y coil }}=-\frac{\mu_{0} I a^{2}}{2\left(a^{2}+\left(y-y_{0}\right)^{2}\right)^{3 / 2}} \hat{y}$

To give the desired field magnitude with a maximum current of 4A per coil, we use coils of 7 layers of 31 turns on the $\mathrm{x}$ - and $\mathrm{z}$ - axes and a coil of 7 layers and 7 turns on the $\mathrm{y}$-axis. Plugging in the dimensions of our trap layout (done in Mathematica in Appendix B.1) to Eq. (23) and Eq. (24), we can express the magnetic field in terms of the currents supplied to each coil.

$$
\vec{B}=10^{-5}\left(\left[\begin{array}{c}
-18.9744 \\
2.4984 \\
0
\end{array}\right] I_{x}+\left[\begin{array}{c}
0 \\
-24.7402 \\
0
\end{array}\right] I_{y}+\left[\begin{array}{c}
0 \\
2.4984 \\
18.9744
\end{array}\right] I_{z}\right)
$$

where each current is in Amperes and we define each current to be positive when it is aligned such that the induced field will point towards the trap center. We can then solve for the necessary currents

$$
\left[\begin{array}{l}
I_{x} \\
I_{y} \\
I_{z}
\end{array}\right]=10^{5}\left[\begin{array}{ccc}
-18.9744 & 0 & 0 \\
2.4984 & -24.7402 & 2.4984 \\
0 & 0 & 18.9744
\end{array}\right]^{-1} \vec{B}_{\text {gen }}
$$

where $\vec{B}_{\text {gen }}$ is the field given by Eq. 21 in Tesla. Explicitly, this gives

$$
\left[\begin{array}{l}
I_{x} \\
I_{y} \\
I_{z}
\end{array}\right]=\left[\begin{array}{ccc}
-5270.3 & 0 & 0 \\
-532.22 & -4042.0 & 532.22 \\
0 & 0 & 5270.3
\end{array}\right]\left[\begin{array}{c}
-B_{x}^{\mathrm{bg}} \\
-B_{y}^{\mathrm{bg}} \\
-B_{z}^{\mathrm{bg}}+B_{z}^{\exp }
\end{array}\right]
$$

According to the National Centers for Environmental Information's World Magnetic Model, the magnetic field of the Earth in Bloomingtion Indiana $\left(39.1653^{\circ} \mathrm{N}, 86.5264^{\circ} \mathrm{W}, 771^{\prime}\right.$ elevation) is

$$
\vec{B}^{\mathrm{bg}}=\left[\begin{array}{l}
2.04 \times 10^{-5} \\
1.56 \times 10^{-5} \\
4.81 \times 10^{-5}
\end{array}\right] \mathrm{T}
$$

When mapping cardinal directions to laboratory defined coordinates $(\hat{x}=\mathrm{S}, \hat{y}=\mathrm{W})$. To cancel this field and generate the necessary field for the experiment of $3.930 \times 10^{-4} \mathrm{~T}$ in $\hat{z}$, we need currents

$$
\left[\begin{array}{l}
I_{x} \\
I_{y} \\
I_{z}
\end{array}\right]=\left[\begin{array}{ccc}
-5270.3 & 0 & 0 \\
-532.22 & -4042.0 & 532.22 \\
0 & 0 & 5270.3
\end{array}\right]\left[\begin{array}{c}
-2.04 \times 10^{-5} \\
-1.56 \times 10^{-5} \\
2.91 \times 10^{-4}
\end{array}\right]=\left[\begin{array}{l}
.1075 \\
1720 \\
1.5337
\end{array}\right] \mathrm{A}
$$

While the actual field produced by the coils is likely to differ from the theoretical calculation for systematic reasons (imperfect winding of coils, dimensions not exactly matching $3 \mathrm{~d}$ modeling, etc.), this calculation will at least put us in a starting point for calibration, which we can do either with a hall sensor or using the trapped ions themselves as probes.

\subsection{Laser Pulse Generation}

We need to produce stable and narrow laser pulses at various wavelengths to drive the atomic transitions of the experiment. Table 1 enumerates these. The Zeeman transition wavelengths come from Eq. 14.

Table 1: A table of required transition frequencies for the experiment

\begin{tabular}{|c|l|}
\hline Frequency & \multicolumn{1}{c|}{ Purpose } \\
\hline$f_{0}-21.16 \mathrm{MHz}$ & $|0\rangle \leftrightarrow|-3\rangle$ transition \\
$f_{0}-21.16 \mathrm{MHz}$ & $|0\rangle \leftrightarrow|-1\rangle$ transition \\
$f_{0}-21.16 \mathrm{MHz}$ & $|0\rangle \leftrightarrow|+1\rangle$ transition \\
$f_{0}-21.16 \mathrm{MHz}$ & $|0\rangle \leftrightarrow|+3\rangle$ transition \\
$811.4 \mathrm{THz}$ & $\mathrm{S}_{1 / 2}-\mathrm{P}_{1 / 2}$ fluorescence pulse \\
\hline
\end{tabular}


Here, $f_{0}$ is the baseline frequency discussed in section 3.3 with value $642121496772645.15(52) \mathrm{Hz}$ [4]. To produce these frequencies we use separate sources for the $811.4 \mathrm{THz}$ and baseline $f_{0}$ beams and use acousto-optic modulators (AOMs) to shift the frequency of the baseline beam to the various order $10 \mathrm{MHz}$ shifts. Fig. 13 depicts a layout for generating and controlling the transition pulses.

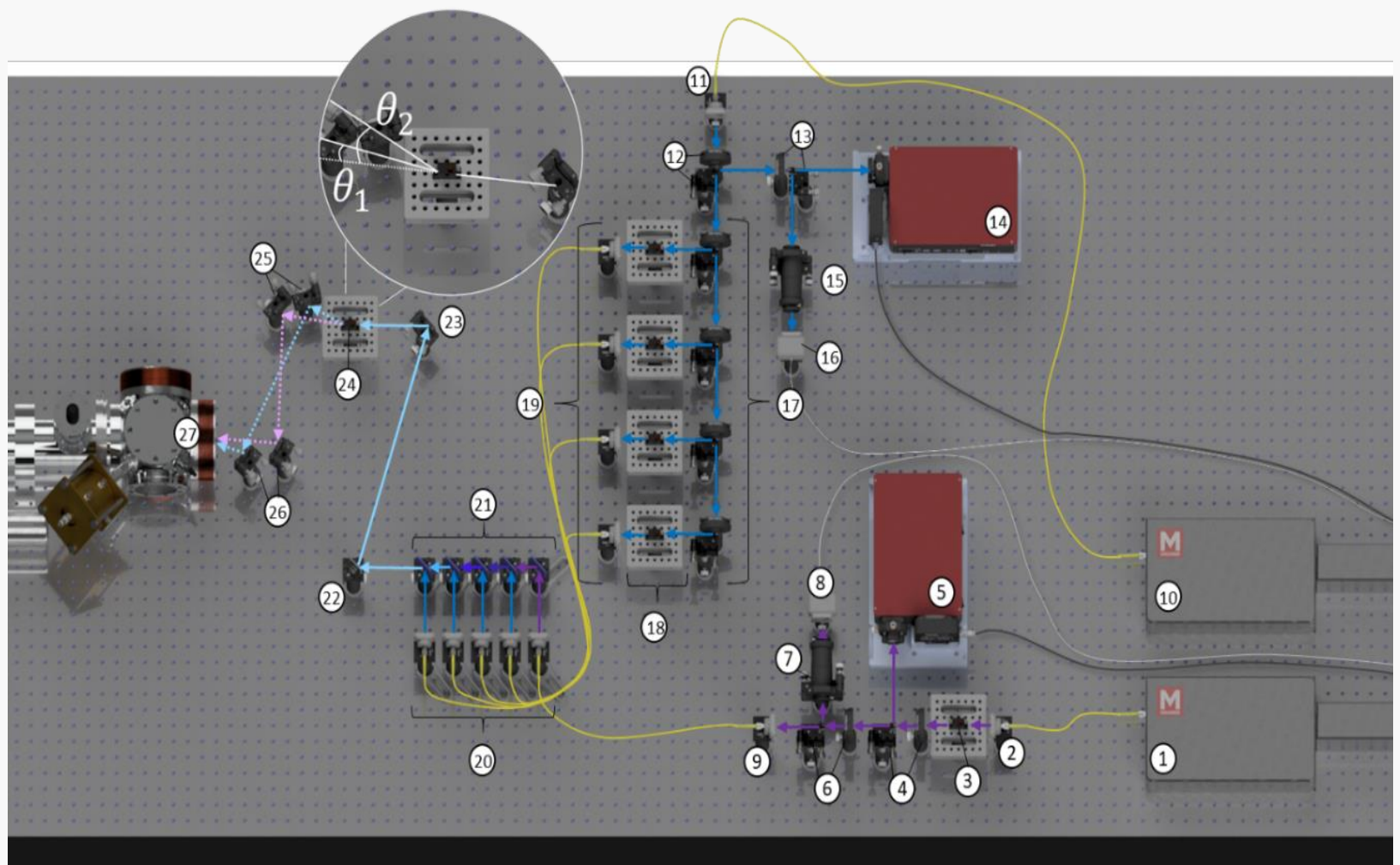

\begin{tabular}{|l|l|l|l|}
\hline Label No. & Component & Label No. & Component \\
\hline 1 & $467 \mathrm{~nm}$ baseline source & 8,16 & Photodetector \\
$2,9,11,19,20$ & Optical fiber coupler & 10 & $369 \mathrm{~nm}$ source \\
3,18 & AOM & 21 & Beam combiner \\
$4,6,12,13,17$ & Polarizer-polarizing beamsplitter pair & $22,23,25,26$ & Mirror \\
& & & \\
5,14 & Wavemeter & 27 & Ion trap window \\
7,15 & Fabry-Pérot cavity & & \\
\hline
\end{tabular}

Figure 13: A diagram of the optical breadboard setup for the creation and application of various laser profiles for preforming atomic transitions

The $881.4 \mathrm{THz}(369 \mathrm{~nm})$ beam is sent through an AOM (No. 3) to allow us to tune the beam and turn the signal on an off quickly according to the desired Rabi period. Then the beam is sent through a polarizerpolarizing beamsplitter pair (No. 4) which allows us to select the intensity of the beam that split off. We partition a small intensity to send to a wavemeter (No. 5) to monitor the frequency. We use a another beamsplitting pair (No. 6) to remove another small portion of the beam and send it through a Fabry-Pérot cavity (No. 7) as discussed in section 3.3. The ouput of the Fabry-Pérot cavity is sent to a photodetector (No. 8) to allow this beampath to function as an interferometer, which will serve as a feedback signal to our laser locking. This way, the wavemeter lets us find our frequency with $\mathrm{MHz}$ precision and the FabryPérot interferometer lets us find and lock the frequency to the single $\mathrm{Hz}$ level. The baseline $f_{0}(467 \mathrm{~nm})$ beam has a small stabilization reference signal split off by (No. 12), which is then split again between a similar wavemeter (No. 14) and Fabry-Perot interferometer (No. 15,16) pair to lock the frequency. The signal is then sent through a series of polarizer-beamsplitter pairs (No. 17) which split the signal into our 
Schlossberger et al., Adv.J. Grad. Res.; Vol. 4, Issue 1, pp: 15-33, July 2018

four $\mathrm{F}_{7 / 2}$ transition beams. Each beam is sent through its own AOM (No. 18) to frequency shift to the appropriate energy. Finally, all the beams are combined in a series of beam combiners (No. 21), then sent through a final AOM (No. 24). By sending a superimposed driving signal to the AOM at two different frequencies, we can create an angular separation $\left(\theta_{1}\right.$ and $\theta_{2}$ in the inset) of the beam into two separate beams whose amplitudes are proportional to the amplitude of each driving frequency. By aiming these separated beams at the two separate ions with a set of mirrors (No. 25,26), we can then control how much of our beam goes to each ion by changing these amplitudes. Because we have an AOM in each beam's path, we can control which beams are applied at any time, and with the final AOM we can control which ion we address. This constitutes a complete control system.

The AOMs require a driving signal whose frequency equals the difference between current and desired laser frequencies. To accomplish this, we can use a Direct Digital Synthesis (DDS) array, which uses a frequency reference, a numerically controlled oscillator, and a digital to analog converter to produce a waveform. This allows us to generate multiple channels specific frequencies on the fly which we can control with LabVIEW using a single reference clock. We can also include in RF switches on each channel so we can control the pulse timing with time resolute TTL logic from an FPGA board. The Monroe group has developed boards based on the Analog Devices AD9912 chip, which we can arrange in a configuration shown in Fig. 14. The system requires a single $1 \mathrm{GHz}$ stable reference clock to provide 12 driving signals.

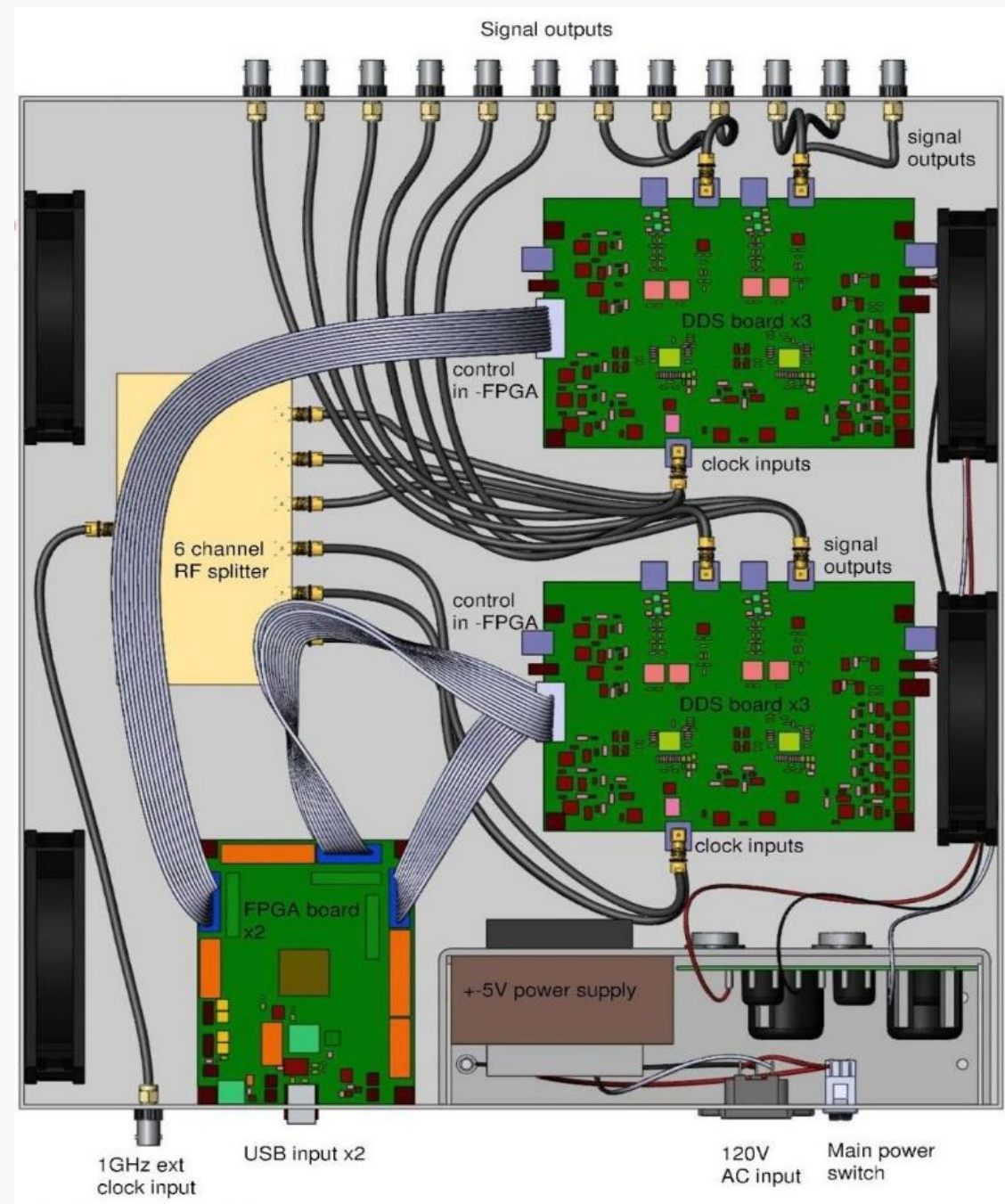

Figure 14: The LabVIEW-controlled DDS board array that produces multiple channels of configurable signal generation 


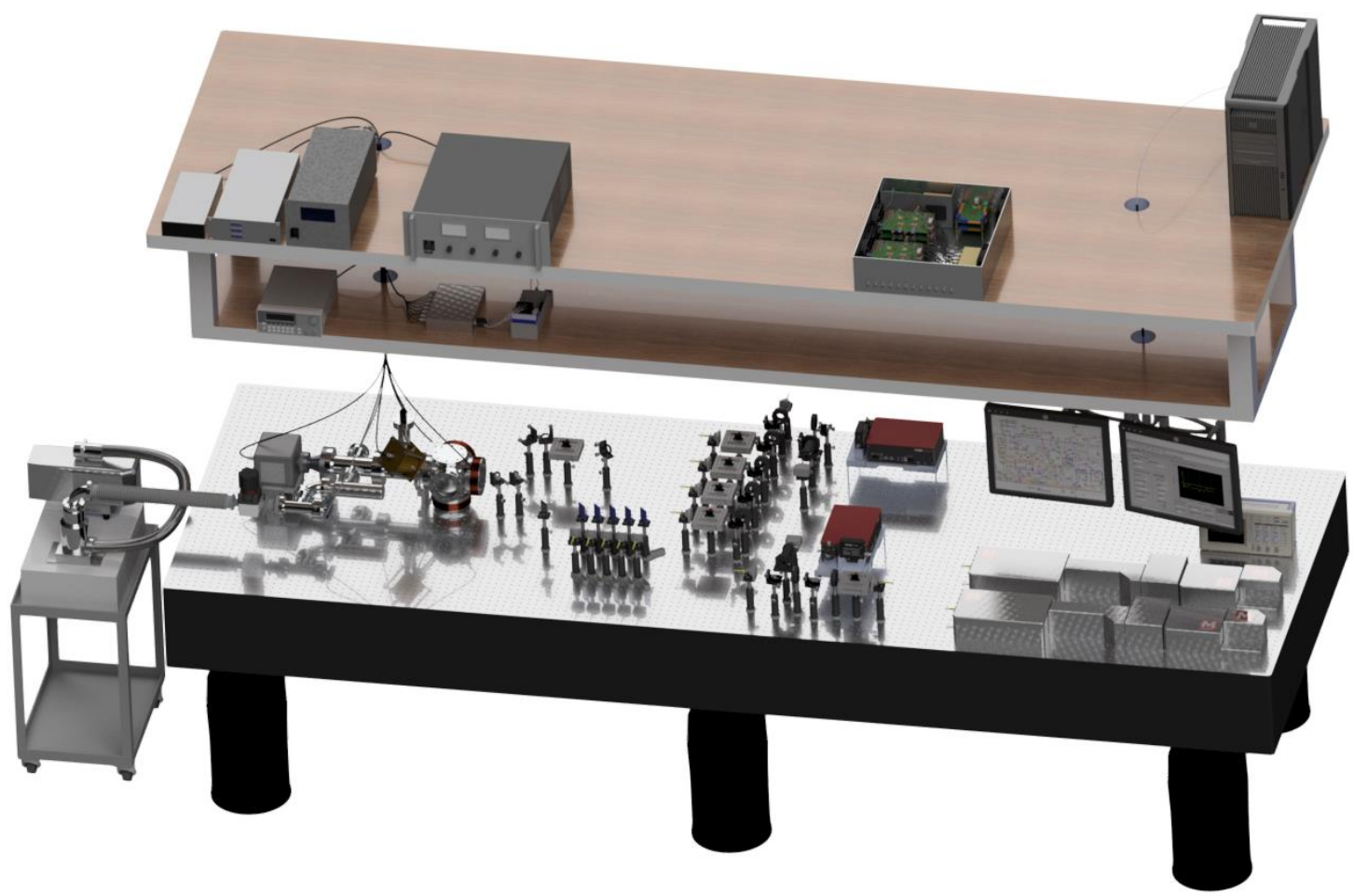

Figure 15: A CAD model of the comprehensive experiment layout

\section{Conclusion}

In this paper we have described a configuration for executing the measurement, including a theoretical description of the experiment, a proposed algorithm for creating, evolving, and measuring the states, and a complete description of the hardware necessary for performing this algorithm. A 3-D model of the complete hardware layout is depicted in Fig. 15. On the bottom left is the Paul trap enclosed in the vacuum system, which itself is enclosed in the array of coils that produce the experiment field. Above this are the electronics that control vacuum components and drive the Paul trap electrodes, including the helical resonator and the DC array. Finally, on the right is the optics system and the experiment control computer running LabVIEW. The whole experiment is mounted on a vibrationally isolated table to provide dimensional self-stability and to prevent vibrational noise from disturbing the experiment.

Although we have techniques for addressing it, the major difficulty of the experiment promises to be executing the $467 \mathrm{~nm}$ transition to initialize the mixed state that decoheres into the measurement state based upon the narrow laser linewidth required and the slow Ramsey oscillation frequency of the transition. Even if we use the $\mathrm{D}_{5 / 2}$ as an intermediary state, the extended lifetime of the $\mathrm{F}_{7 / 2}$ state (the same characteristic we utilize to increase precision) implies that a narrow energy will be required to drive the transition, so there will inherently be issues in reliably getting to this state. However, should we overcome this issue it, should be possible to decrease the bounds on the LLI violation matrix element to as low as $10^{-23}$, a decrease of current bounds by five orders of magnitude.

\section{Acknowledgments}

We would like to give thanks to Yuanheng Xie for his collaboration on the design and calculations regarding the field-inducing coil.

\section{How to Cite this Article:}

N. Schlossberger and P. Richerme, “An Experimental Configuration to Probe for Lorentz Symmetry Violation in Electrons Using Trapped $\mathrm{Yb}+$ Ions”, Advanced Journal of Graduate Research, vol. 4, no. 1, pp. 15-33, Apr. 2018. doi:10.21467/ajgr.4.1.15-33 
Schlossberger et al., Adv.J. Grad. Res.; Vol. 4, Issue 1, pp: 15-33, July 2018

\section{Appendices}

Appendix A.1 \& Appendix B.1: Available at URL https://journals.aijr.in/index.php/ajgr/article/view/447/141

\section{References}

[1] T. Pruttivarasin et al., "Michelson-Morley analogue for electrons using trapped ions to test Lorentz symmetry," Nature, vol. 517, no. 7536, pp. 592-595, Jan. 2015.

[2] V. A. Dzuba et al., "Strongly enhanced effects of Lorentz symmetry violation in entangled Yb+ ions," Nat. Phys., vol. 12, no. 5, pp. 465-468, Jan. 2016.

[3] C. J. Foot, Atomic physics. Oxford: Oxford University Press, 2005.

[4] N. Huntemann, M. Okhapkin, B. Lipphardt, S. Weyers, C. Tamm, and E. Peik, "High-Accuracy Optical Clock Based on the Octupole Transition in Yb + 171," Phys. Rev. Lett., vol. 108, no. 9, p. 90801, Feb. 2012.

[5] M. Roberts, P. Taylor, G. P. Barwood, W. R. C. Rowley, and P. Gill, "Observation of the ${ }^{2} \mathrm{~S}_{1 / 2}{ }^{2} \mathrm{~F}_{7 / 2}$ electric octupole transition in a single ${ }^{171} \mathrm{Yb}^{+}$ion," Phys. Rev. A, vol. 62, no. 2, p. 20501, Jul. 2000.

[6] E. Biémont and P. Quinet, "Theoretical Study of the $4 \mathrm{f}^{14} 6 \mathrm{~s}^{2} \mathrm{~S}_{1 / 2}-4 \mathrm{f}^{13} 6 \mathrm{~s}^{2}{ }^{2} \mathrm{~F}_{7 / 2}^{0} \mathrm{E} 3$ Transition in Yb II," Phys. Rev. Lett., vol. 81, no. 16, pp. 3345-3346, Oct. 1998.

[7] P. Taylor, M. Roberts, G. M. Macfarlane, G. P. Barwood, W. R. C. Rowley, and P. Gill, "Measurement of the infrared 2F $7 / 2-2 D_{5 / 2}$ transition in a single $171 \mathrm{Yb}+$ ion," Phys. Rev. A, vol. 60, no. 4, pp. 2829-2833, Oct. 1999.

[8] D. J. Wineland, C. Monroe, W. M. Itano, D. Leibfried, B. E. King, and D. M. Meekhof, "Experimental Issues in Coherent QuantumState Manipulation of Trapped Atomic Ions," J. Res. Natl. Inst. Stand. Technol. J. Res. Natl. Inst. Stand. Technol, vol. 103 , no. 103.

[9] J. D. Siverns, L. R. Simkins, S. Weidt, and W. K. Hensinger, "On the application of radio frequency voltages to ion traps via helical resonators," Appl. Phys. B, vol. 107, no. 4, pp. 921-934, Jun. 2012.

[10] J. D. Jackson, Classical electrodynamics. Wiley, 1999.

Publish your research article in AIJR journals-

$\checkmark$ Online Submission and Tracking

$\checkmark$ Peer-Reviewed

$\checkmark$ Rapid decision

$\checkmark$ Immediate Publication after acceptance

$\checkmark \quad$ Articles freely available online

$\checkmark \quad$ Retain full copyright of your article.

Submit your article at journals.aijr.in
Publish your books with AIJR publisher-

$\checkmark$ Publish with ISBN and DOI.

$\checkmark$ Publish Thesis/Dissertation as Monograph.

$\checkmark$ Publish Book Monograph.

$\checkmark$ Publish Edited Volume/ Book.

$\checkmark$ Publish Conference Proceedings

$\checkmark \quad$ Retain full copyright of your books.

Submit your manuscript at books.aijr.org 